\title{
A New Family of Explicit Time Integration Algorithms with Controllable Accuracy for Structural Dynamics
}

\author{
Xiaoqiong Du, a , Yongliang Zhao ${ }^{1, b}$, Xiaoliang Yan ${ }^{1, c}$ \\ ${ }^{1}$ Aviation Equipment Research Institute, AVIC Qing-an Group Co., Ltd, Xi'an, 710077, China \\ axqdu0101@163.com, bylzhao@163.com, cyanxiaoliang111@126.com
}

Keywords: Structural Dynamics, Explicit Time Integration Algorithm, Controllable Accuracy, Algorithm Design

\begin{abstract}
A new family of explicit time integration algorithms with controllable accuracy for structural dynamics are proposed in this paper based on discrete control theory. Firstly, the transfer function of the new algorithms with integration coefficients are obtained by $Z$ transform, then the coefficient expressions of the new algorithms are derived according to the poles condition. The theoretical analyses about the stability and accuracy indicate that the new algorithms possess the properties of second-order accuracy, zero amplitude decaying, moreover, the period elongation can be controlled by parameter $\lambda$, which can adjust the accuracy of the new algorithms. When $\lambda$ is less than or equal to 4 , the algorithm are unconditionally stable for the linear system.
\end{abstract}

\section{Introduction}

Time integration algorithms are typically utilized to solve the motion equation in structural dynamics. Various methods have been used to develop time integration algorithms, such as Taylor formula expansion, weighted residual method, least squares method and Hamiltonian principle. The existing algorithms are mainly divided into explicit and implicit algorithm. If the structural displacement for the next time step can be determined from responses of current and previous time step, the algorithm is explicit, otherwise it is implicit. Explicit algorithms are usually conditionally stable and do not require iterative solution, e.g., the central difference method. Implicit algorithms are usually unconditionally stable and require to solve simultaneous equations, such as Newmark method [1], HHT- $\alpha$ method [2], WBZ- $\alpha$ method [3], generalized- $\alpha$ method [4]. For the structures with a large number of DOFs (degrees of freedom), the explicit algorithms can greatly improve computational efficiency.

Recently, due to the need for fast and accurate dynamical simulation of pseudo-dynamic testing and real-time hybrid testing [5], unconditionally stable explicit algorithms are paid close attention. Chang proposed a series of unconditionally stable explicit algorithms [6-8], transformed the stability of the explicit algorithms from the traditional conditionally stable to unconditionally stable. Based on discrete control theory, Chen and Ricles proposed an unconditionally stable CR explicit algorithm, clarified the whole process of algorithm design [9]. Gui proposed an unconditionally stable Gui- $\lambda$ explicit algorithm, and parameter $\lambda$ can adjust the accuracy of algorithm, CR algorithm is a special case of Gui- $\lambda$ algorithm [10]. Kolay and Ricles combined the equilibrium equation of the generalized- $\alpha$ method with the displacement and velocity recursion of CR algorithm, designed an unconditionally stable explicit KR- $\alpha$ algorithm with numerical dissipation [11].

As a new method of time integration algorithm design, discrete control theory makes the algorithm derivation and analysis more rational and convenient. Based on the discrete control theory, this paper assumes the displacement and velocity recursion, making the new algorithms with unknown coefficients have same poles as the Gui- $\lambda$ algorithm, thus a new family of explicit time integration algorithms for structural dynamics are designed. Then theoretical analyses about the stability of linear system, truncation error, amplitude decaying and period elongation of the new algorithm are conducted. 


\section{Design of the Explicit Time Integration Algorithms}

The Gui- $\lambda$ algorithm can effectively be used to solve the approximate solution of motion equation for structural dynamics, which is described by the following three equations [10]:

$$
\begin{aligned}
& m \ddot{x}_{i+1}+c \dot{x}_{i+1}+k x_{i+1}=f_{i+1} \\
& \dot{x}_{i+1}=\dot{x}_{i}+\alpha_{1} h \ddot{x}_{i} \\
& x_{i+1}=x_{i}+h \dot{x}_{i}+\alpha_{2} h^{2} \ddot{x}_{i}
\end{aligned}
$$

where,

$$
\alpha_{1}=\alpha_{2}=\lambda /\left(\lambda+\lambda \Omega \xi+\Omega^{2}\right)
$$

Eqs. (1), (2) and (3) represent the equilibrium equation, recursive formula of velocity and displacement of Gui- $\lambda$ algorithm for SDOF system separately; where $m, c=2 m \omega \xi, k=m \omega^{2}$ are the mass, damping and stiffness, respectively; $\omega$ is the natural frequency; $\xi$ is equal to the damping ratio; $\ddot{x}_{i}, \dot{x}_{i}, x_{i}$ and $f_{i}$ stand for acceleration, velocity, displacement and external force at $i$ th time step; $h$ denotes the integration step; $\alpha_{1}$ and $\alpha_{2}$ are integration coefficients. The new explicit time integration algorithms with controllable accuracy are designed based on Gui- $\lambda$ algorithm.

Generally, two kinds of methods are used to analyze the property of time integration algorithms [11]. The first one is to use the eigenvalues of amplification matrix [12]. The algorithm can be represented as

$$
X_{i+1}=A X_{i}, X_{i}=\left[x_{i}, h \dot{x}, h^{2} \ddot{x}_{i}\right]^{T}
$$

where, $A$ is the amplification matrix of algorithm. The second one is to use discrete control theory to solve the poles of the transfer function. It is worth noting that the poles of the transfer function are equivalent to the eigenvalues of the amplification matrix $[9,11]$. Compared with the eigenvalues, solving the poles of transfer function is more simple and convenient. This paper adopts the latter method to analyze the property of the new algorithms.

The transfer function of system is the ratio of the $Z$ transform of the response to the $Z$ transform of the input. The definition and property of the $Z$ transform are [13]

$$
Z\left(F_{j}\right)=F(z), Z\left(F_{j-1}\right)=z^{-1} F(z)
$$

where, $Z\left(F_{j}\right)$ stands for the $Z$ transform of $F(t)$ at $i$ th time step. Therefore, the transfer function $G(z)$ of Gui- $\lambda$ algorithm is

$$
G(z)=\frac{X(z)}{F(z)}=\frac{n_{3} z^{3}+n_{2} z^{2}+n_{1} z+n_{0}}{d_{3} z^{3}+d_{2} z^{2}+d_{1} z+d_{0}}
$$

The corresponding open-loop block diagram is shown in Fig. 1.

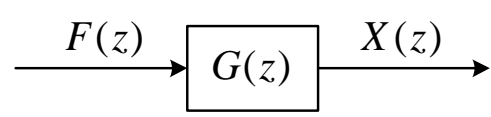

Fig. 1 Block diagram of open-loop system.

where, $X(z)$ and $F(z)$ are the $Z$ transform of displacement and external force, respectively. The coefficients of $G(z)$ are shown in Table 1 , in which $\Omega=\omega h$.

Table 1. Coefficients of transfer function $G(z)$ for Gui- $\lambda$ algorithm.

\begin{tabular}{cccccc}
\hline$n_{2}$ & $n_{1}$ & $n_{0}$ & $d_{2}$ & $d_{1}$ & $d_{0}$ \\
\hline 0 & $\alpha_{2} h^{2}$ & $\left(\alpha_{1}-\alpha_{2}\right) h^{2}$ & $m$ & $\left(\alpha_{2} \Omega^{2}+2 \alpha_{1} \Omega \xi-2\right) m$ & $\left\{\left(\alpha_{1}-\alpha_{2}\right) \Omega^{2}-2 \alpha_{1} \Omega \xi+1\right\} m$ \\
\hline
\end{tabular}


The characteristic equation, which makes the denominator of transfer function $G(z)$ be equal to zero, is represented by

$$
d_{2} z^{2}+d_{1} z+d_{0}=0
$$

The roots of the characteristic equation are the poles of the open-loop system.

Assuming the recursive formula of velocity and displacement for the new algorithms are described by the following equations:

$$
\begin{aligned}
& \dot{x}_{i+1}=\dot{x}_{i}+\beta_{1} h\left(\ddot{x}_{i}-\ddot{x}_{i-1}\right) \\
& \quad x_{i+1}=x_{i}+h \dot{x}_{i}+\beta_{2} h^{2}\left(\ddot{x}_{i}-\ddot{x}_{i-1}\right)
\end{aligned}
$$

where, $\beta_{1}$ and $\beta_{2}$ are the unknown coefficients, the transfer function $G^{\prime}(z)$ of the new algorithms is

$$
G^{\prime}(z)=\frac{X(z)}{F(z)}=\frac{n_{3}^{\prime} z^{3}+n_{2}^{\prime} z^{2}+n_{1}^{\prime} z+n_{0}^{\prime}}{d_{3}^{\prime} z^{3}+d_{2}^{\prime} z^{2}+d_{1}^{\prime} z+d_{0}^{\prime}}
$$

The coefficients of $G^{\prime}(z)$ are shown in Table 2 .

Table 2. Coefficients of transfer function $\mathrm{G}^{\prime}(\mathrm{z})$ for the new algorithms.

\begin{tabular}{cccccc}
\hline$n_{2}^{\prime}$ & $n_{1}^{\prime}$ & $n_{0}^{\prime}$ & $d_{2}^{\prime}$ & $d_{1}^{\prime}$ & $d_{0}^{\prime}$ \\
\hline 0 & $\beta_{2} h^{2}$ & $\left(\beta_{1}-\beta_{2}\right) h^{2}$ & $m$ & $\left(\beta_{2} \Omega^{2}+2 \beta_{1} \Omega \xi-1\right) m$ & $\left\{\left(\beta_{1}-\beta_{2}\right) \Omega^{2}-2 \beta_{1} \Omega \xi\right\} m$ \\
\hline
\end{tabular}

The stability, amplitude decaying and period elongation of the algorithm are related to the poles of the transfer function [11]. If two algorithms have same poles, they will have same stability, amplitude decaying and period elongation. Based on this, it is assumed that the new algorithm has the same pole as the Gui- $\lambda$ algorithm, namely, satisfying the following relationship

$$
d^{\prime}{ }_{1}=d^{\prime}{ }_{2} d_{1} / d_{2}, d^{\prime}{ }_{0}=d^{\prime}{ }_{2} d_{0} / d_{2}
$$

Thus the unknown coefficients $\beta_{1}$ and $\beta_{2}$ can be solved

$$
\beta_{1}=\frac{\lambda}{\Omega^{2}+\lambda \xi \Omega+\lambda}, \beta_{2}=\frac{(\lambda-1) \Omega^{2}-\lambda \xi \Omega-\lambda}{\Omega^{2}\left(\Omega^{2}+\lambda \xi \Omega+\lambda\right)}
$$

Therefore, a new family of explicit algorithms with controllable accuracy are proposed, described by Eq. (1), (9) and (10). The stability and accuracy of the new algorithms will be analyzed in the following setions.

\section{Stability Analysis of the New Algorithms}

For any value $\Omega \in[0, \infty]$, if the poles of open-loop transfer function are located within or on the unit circle in $z$-domain, then the system is stable, which is consistent with that the spectral radius is less than or equal to one. The poles $p_{1,2}$ can be finally expressed as the function of $\Omega$ and $\lambda$ for undamped case.

$$
p_{1,2}=P \pm Q i
$$

where,

$$
P=\frac{2 \Omega^{2} / \lambda-\Omega^{2}+2}{2\left(1+\Omega^{2} / \lambda\right)}, Q=\frac{\Omega \sqrt{4 \Omega^{2} / \lambda-\Omega^{2}+4}}{2\left(1+\Omega^{2} / \lambda\right)}
$$

In order to make the expression $Q$ meaningful, $\Omega$ and $\lambda$ need to meet the following equations:

$\lambda \leq 4 \quad 0<\Omega<\infty$ 


$$
\lambda>4 \quad \Omega^{2} \leq 4 \lambda /(\lambda-4)
$$

After calculating, $P^{2}+Q^{2}=1$, so the spectral radius $\rho=\max \left\{\left|p_{i}\right|\right\}(i=1,2)$ is equal to 1 . Thus, when $\lambda \leq 4$, the algorithms are unconditionally stable for linear system. When $\lambda>4$, the algorithms are conditionally stable, as $\lambda$ increases, the stability range of $\Omega$ decreases, $\Omega$ and $\lambda$ need to satisfy Eq. (17).

\section{Accuracy Analysis of the New Algorithms}

Local truncation error, amplitude decaying and period elongation are often used to investigate the accuracy of the algorithms [13]. They will be discussed separately hereafter.

Local Truncation Error. The high-order small quantity, omitted in the approximate expression of acceleration, velocity and displacement of the time integration algorithms, can be estimated by the local truncation error $\tau$, which is generally defined as

$$
\tau=h^{-2}\left[x(i+1)-A_{1} x(i)+A_{2} x(i-1)-A_{3} x(i-2)\right]
$$

where, $A_{1}=$ trace of $A, A_{2}=$ sum of principal minors of $A, A_{3}=$ determinant of $A, A$ is the amplification matrix, as shown in Eq.(5). An algorithm is $k$ th order provided that $\tau=o\left(h^{k}\right)$ [4]. By expanding $x(i+1)$, $x(i-1), x(i-2)$ as Taylor series at $i$ th time step, and combing with the equilibrium Eq. (1) at $i$ th time step, local truncation error $\tau$ of the new algorithms is formulated by

$$
\tau=\left(-\omega^{2}+\beta_{1} \omega^{2}+\beta_{1} \omega^{3} \xi h\right) x_{i}+\left(-2 \omega \xi+2 \beta_{1} \omega \xi+2 \beta_{1} \omega^{2} \xi^{2} h\right) \dot{x}_{i}+o\left(h^{2}\right)
$$

As the time step $h$ tends to zero, the limit of $\tau$ is $o\left(h^{2}\right)$, thus the new algorithms have second-order accuracy.

Amplitude Decaying and Period Elongation. The accuracy of the algorithms can also be measured by the amplitude decaying $A D$ and the period elongation $P E$. The open-loop poles of the new explicit algorithms can be expressed in the following form

$$
p_{1,2}=P \pm Q i=\exp \left[\bar{\Omega}\left(-\bar{\xi} \pm i \sqrt{1-\bar{\xi}^{2}}\right)\right]
$$

where, $P$ and $Q$ are shown as Eq. (15), $\bar{\xi}=-\ln \left(P^{2}+Q^{2}\right) / 2 \bar{\Omega}, \quad \bar{\Omega}=\tan ^{-1}(Q / P) / \sqrt{1-\bar{\xi}^{2}}$. The amplitude decaying and the period elongation are defined as

$$
A D=1-\exp (-2 \pi \bar{\xi}), \quad P E=\Omega / \bar{\Omega}-1
$$

After simple calculation, $A D=0$, therefore, there is no decay of response amplitude for the new algorithms.

The period elongation $P E$ is a function of the variables $\lambda$ and $\Omega$. Fig. 2 demonstrate the $P E$ for the new algorithms with different values of $\lambda$, where NCAA is the Newmark constant acceleration algorithm.

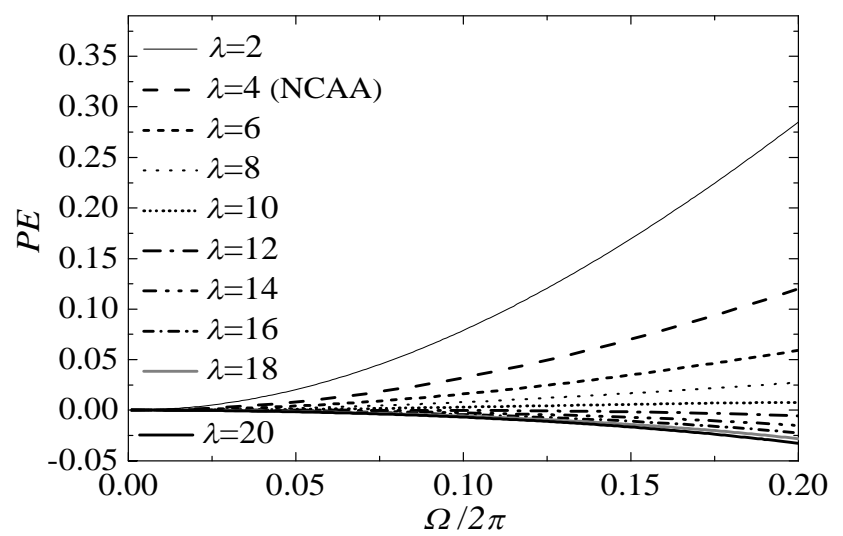

Fig. $2 P E$ values corresponding to $\lambda$ with $2 \sim 20$. 
Fig. 2 shows that the period elongation $P E$ is closely related to the parameter $\lambda$, with the increase of $\lambda$, the absolute value of $P E$ decreases first and then increases for fixed value of $\Omega$, therefore, the accuracy of the new algorithm can be controlled by $\lambda$. When $\lambda$ takes the value of $10 \sim 12$, the absolute value of $P E$ for the new algorithms is significantly smaller than the $P E$ value of NCAA, thus the new algorithms have higher accuracy with $\lambda$ between $10 \sim 12$.

\section{Conclusion}

In this paper, a new family of explicit time integration algorithms for structural dynamics are proposed, which have the merits of second-order accuracy, zero amplitude decaying and controllable period elongation. Parameter $\lambda$ can control the accuracy of the new algorithms by adjusting the period elongation, with the increase of $\lambda$, the accuracy of the algorithm increases first and then decreases. The parameter $\lambda$ can also control the stability range of the algorithms. As $\lambda$ increases, the stability range decreases. For the linear system, when $\lambda \leq 4$, the new algorithms are unconditionally stable. If $\lambda>4$, the new algorithms are conditionally stable.

\section{References}

[1] N. Newmark. A method of computation for structural dynamics, Proceedings of the ASCE. J. Eng. Mech. Div. 85(3) (1959) 67-94.

[2] H. M. Hilber, T. J. R. Hughes, R. L. Taylor. Improved numerical dissipation for time integration algorithms in structural dynamics. Earthq. Eng. Struct. Dyn. 5(3) (1977) 283-292.

[3] W. L. Wood, M. Bossak, O. C. Zienkiwicz. An alpha modification of Newmark's method. Int. J. Num. Meth. Eng. 15 (1981) 1562-1566.

[4] J. Chung, G. M. Hulbert. A time integration algorithm for structural dynamics with improved numerical dissipation: the generalized- $\alpha$ method. J. Appl. Mech. (ASME), 60 (1993) 371-375.

[5] G. Ou, A. I. Ozdagli, S. J. Dyke, B. Wu. Robust integrated actuator control: experimental verification and real-time hybrid-simulation implementation. Earthq. Eng. Struct. Dyn. 44 (2015) 441-460.

[6] S. Y. Chang. Explicit pseudo dynamic algorithm with unconditional stability. J. Eng. Mech. 128(9) (2002) 935-947.

[7] S. Y. Chang. An explicit method with improved stability property. Int. J. Num. Meth. Eng. 77 (2009) 1100-1120.

[8] S. Y. Chang. Family of structure-dependent explicit method for structural dynamics. J. Eng. Mech. 140 (2014) 06014005.

[9] C. Chen, J. M. Ricles. Development of direct integration algorithms for structural dynamics using discrete control theory. J. Eng. Mech. 134(8) (2008) 676-683.

[10] Y. Gui, J. T. Wang, F. Jin, C. Chen, M. X. Zhou. Development of a family of explicit algorithms for structural dynamics with unconditional stability. Nonlin. Dyn. 77(4) (2014) 1157-1170.

[11] C. Kolay, J. M. Ricles. Development of a family of unconditionally stable explicit direct integration algorithms with controllable numerical energy dissipation. Earthq. Eng. Struct. Dyn. 43(9) (2014) 1361-1380.

[12] G. M. Hulbert. Computational structural dynamics. Encycloped. Comput. Mech. Vol. 2: Solids and Structures, John Wiley \& Sons, New York, 2004.

[13] C. S. Liu, Q. X. Wu. Fundamentals of Modern Control Engineering. Beijing: Science Press, 
ATLANTIS
PRESS

2011. 